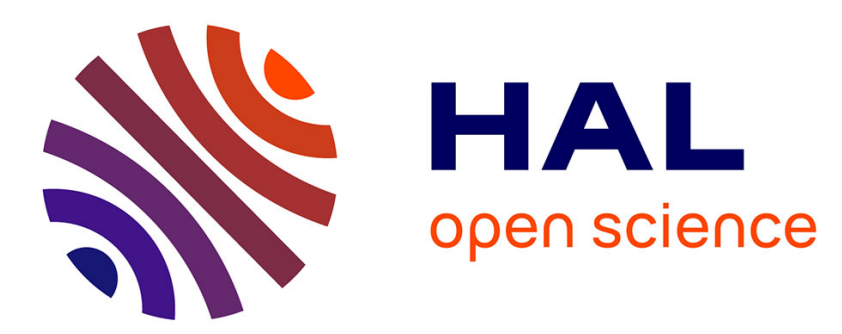

\title{
Vessel probe CT protocol in the study of esophageal carcinoma: can it improve preoperative $\mathrm{T}$ staging?
}

M. Moschetta, A.A. Stabile Ianora, A. Marzullo, A. Scardapane, G. Angelelli

\section{To cite this version:}

M. Moschetta, A.A. Stabile Ianora, A. Marzullo, A. Scardapane, G. Angelelli. Vessel probe CT protocol in the study of esophageal carcinoma: can it improve preoperative T staging?. EJSO European Journal of Surgical Oncology, 2010, 36 (7), pp.663. 10.1016/j.ejso.2010.05.011. hal00603545

\section{HAL Id: hal-00603545 https://hal.science/hal-00603545}

Submitted on 26 Jun 2011

HAL is a multi-disciplinary open access archive for the deposit and dissemination of scientific research documents, whether they are published or not. The documents may come from teaching and research institutions in France or abroad, or from public or private research centers.
L'archive ouverte pluridisciplinaire HAL, est destinée au dépôt et à la diffusion de documents scientifiques de niveau recherche, publiés ou non, émanant des établissements d'enseignement et de recherche français ou étrangers, des laboratoires publics ou privés. 


\section{Accepted Manuscript}

Title: Vessel probe CT protocol in the study of esophageal carcinoma: can it improve preoperative T staging?

Authors: M. Moschetta, A.A. Stabile lanora, A. Marzullo, A. Scardapane, G. Angelelli

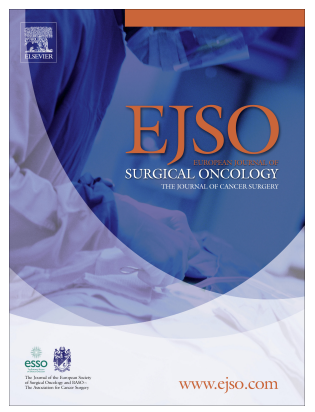

PII:

S0748-7983(10)00119-8

DOI:

10.1016/j.ejso.2010.05.011

Reference: YEJSO 2973

To appear in: European Journal of Surgical Oncology

Received Date: 16 October 2009

Revised Date: 4 March 2010

Accepted Date: 4 May 2010

Please cite this article as: Moschetta M, Stabile lanora AA, Marzullo A, Scardapane A, Angelelli G.

Vessel probe CT protocol in the study of esophageal carcinoma: can it improve preoperative T staging?, European Journal of Surgical Oncology (2010), doi: 10.1016/j.ejso.2010.05.011

This is a PDF file of an unedited manuscript that has been accepted for publication. As a service to our customers we are providing this early version of the manuscript. The manuscript will undergo copyediting, typesetting, and review of the resulting proof before it is published in its final form. Please note that during the production process errors may be discovered which could affect the content, and all legal disclaimers that apply to the journal pertain. 


\section{Vessel probe CT protocol in the study of esophageal carcinoma: can it improve preoperative}

\section{T staging?}

Moschetta $\mathrm{M}^{1}$, Stabile Ianora $\mathrm{AA}^{1}$, Marzullo $\mathrm{A}^{2}$, Scardapane $\mathrm{A}^{1}$, Angelelli $\mathrm{G}^{1}$

${ }^{1}$ Department of Radiology, University of Bari Medical School, Piazza Giulio Cesare 11, 70124

Bari, Italy

${ }^{2}$ Department of Pathological Anatomy, University of Bari Medical School, Piazza Giulio Cesare 11, 70124 Bari, Italy

Correspondence to: Moschetta Marco, Department of Radiology, University of Bari Medical

School, Piazza Giulio Cesare 11, 70124 Bari - Italy

Tel.: 00390805478840; Fax: 00390805592911; e-mail: marco.moschetta@ gmail.com 


\title{
Vessel probe CT protocol in the study of esophageal carcinoma: can it improve preoperative
}

\section{T staging?}

\begin{abstract}
Aims: This study aims to compare transverse images and vessel probe (VP) in MPR mode reconstructions obtained by 16-row MDCT with the histological findings in the preoperative $\mathrm{T}$ staging of esophageal cancer.

Materials and methods: Thirty-one patients (23 M, 8 F, mean age 63.2) with endoscopic and histological diagnosis of esophageal carcinoma underwent CT examination. Esophageal lumen was distended by $\mathrm{CO}_{2}$ and a biphasic technique with 35 s and 70 s delay was used after intravenous injection of contrast material. Transverse and VP in MPR mode images were evaluated and the following parameters were considered: presence and location of the tumor; esophageal wall thickness and enhancement; depth of visceral wall invasion; periesophageal fat morphology and infiltration of adjacent organs. Preoperative staging was performed and then it was compared with the histological findings considered as reference standard.
\end{abstract}

Results: Sensibility, negative predictive and accuracy values were $67 \%, 64 \%$ and $79 \%$ by using axial images for preoperative $\mathrm{T}$ staging, while the use of $\mathrm{VP}$ increased the previous values up to $83 \%, 78 \%$ and $89 \%$, respectively.

Conclusions: In the preoperative staging of esophageal cancer, VP in MPR mode reconstructions obtained by 16-row MDCT increase the sensibility and diagnostic accuracy values in the T parameter evaluation compared with axial images.

Keywords: Esophageal cancer - MDCT - Computed tomography - Staging. 


\section{Introduction}

Esophageal carcinoma is relatively rare and represents the ninth most common cancer in industrialized countries, with an incidence of 3-4/100 000 persons. In Europe and United States it represents approximatively $1 \%$ of all malignancies and the sixth leading cause of death related to cancer $[1,2]$. About $90 \%$ of esophageal tumors consist of carcinomas, with a 40-60\% incidence for squamous cell forms (SCC) and 30-50\% for adenocarcinomas of the esophago-gastric junction (AEG), with the latest representing $80 \%$ of tumors arising from Barrett's esophagus $[3,4]$. The prognosis of patients with esophageal cancer depends on the stage of disease at the time of diagnosis. An accurate evaluation of both depth of esophageal wall invasion and lymph node and distant organ involvement is crucial for choosing an optimal therapeutic strategy, also considering the possibility of preoperative chemo-radiotherapy and individualized treatments. Surgery remains the main therapeutic option and accurate preoperative staging is important to increase the survival of these patients [5]. For the staging of esophageal cancer, endoscopy is used to detect visceral wall abnormalities. At the moment, endoscopic ultrasonography (EUS) is the most common way of evaluating the $\mathrm{T}$ parameter; however, this technique is invasive [6]. Computed tomography (CT) yields useful information on tumor location, size, extension and surgical resectability. The depth evaluation of mural invasion is also improved with clinical application of multidetector CT (MDCT) and multiplanar reconstructions (MPR) [6]. As far as we know, no study has evaluated vessel probe (VP) reconstructions in the T staging of esophageal carcinoma, although it has been recently performed in the gastric cancer staging as a rapid and automatic software which generates accurate imaging of the gastric wall layers in the lesion site with high spatial and contrast resolution and simultaneously in multiple planes [7]. The aim of this study is to evaluate the diagnostic accuracy of 16-row MDCT in the preoperative T staging of esophageal cancer and to compare T staging findings obtained by transverse images and VP in MPR mode reconstructions with histology. 


\section{Materials and methods}

\section{Patients}

Between April 2007 and August 2009, 31 patients (23 men and 8 women, aged 42-75 years, mean age 63.2 years) with endoscopic diagnosis and histologically proven esophageal cancer underwent 16-row MDCT examination 7 days after conventional endoscopy for preoperative staging. This study was approved by the institutional review board and written informed consent was obtained from all patients according to the Declaration of Helsinki principles. Tumor was located on the upper third $(n=3)$, middle third $(n=15)$ or lower third $(n=13)$. No cases of distant metastases occurred. Within two weeks of MDCT examination, 19 patients underwent surgical resection (esophagectomy). The resected organ was histologically evaluated to determine tumor penetration and depth and pathological findings served as the reference standard. Twelve patients affected by advanced cancer were treated with chemo-radiotherapy and subsequently with palliative esophageal stenting because of local extension of the disease. In these last patients, definitive diagnosis was based on histological examination on needle biopsies during endoscopy, CT and EUS findings and broncoscopy. In all cases histology confirmed the malignant nature of the lesions: 18 cases proved to be SCCs and the remaining 13 were AEGs. Histological T staging was based on the international TNM classification as defined by the American Joint Committee on Cancer, shown in Table 1 [8].

\section{CT protocol}

All studies were performed using 16-slice CT (TSX-101A-Aquilion, Toshiba Medical System, Tochigi, Japan). Unenhanced MDCT was not performed. Shortly before CT scanning (approximatively $20 \mathrm{~s}$ ) patients ingested $7 \mathrm{~g}$ of effervescent granules (Duogas, Bracco, Milan, Italy) that generate $\mathrm{CO} 2$ with a small amount of water. We did not administer any antiperistaltic drug and an optimal esophageal distension was reached and well tolerated in all cases. Contrast-enhanced MDCT images were obtained after intravenous injection of iodinated contrast material (Iomeron 400; Bracco, Milan, Italy) into a cubital vein using a 16-18 G needle and an automatic injector 
(MK-IV, Medrad, Pittsburgh, PA) at a dose of $1.5 \mathrm{ml} / \mathrm{kg}$ body weight and injection rate of 3.5 $\mathrm{ml} / \mathrm{s}$. A biphasic technique was used, with $\mathrm{CT}$ acquisition performed in the arterial and portal venous phases (mean start delay of $35 \mathrm{~s}$ for the arterial and $70 \mathrm{~s}$ for the portal phase) with the following parameters: slice thickness $1 \mathrm{~mm}$; increment $0.8 \mathrm{~mm}$; pitch 1.75 ; rotation time $0.5 \mathrm{~s}$; 120/250 KVp/mAs. CT images were obtained with patients in the supine position during a single breath hold in full inspiration. CT data were acquired from neck to lower pole of the kidneys in the arterial phase and from neck to the pubic symphysis in the portal phase, for complete tumor staging. The entire procedure was performed within 15 minutes. All MDCT data were transferred to a workstation (HP XW 6400) equipped with dedicated software (Vitrea 4.1, Vital Images, Minneapolis, MN) for image reconstructions. The post-processing programme used was VP in MPR mode. Arterial phase was used to evaluate the $\mathrm{T}$ staging in order to take advantage of the maximum enhancement of the esophageal wall, which is crucial to use VP software. VP is a programme that allows vessels to be simultaneously examined in $3 \mathrm{D}$, curved reformat and crosssectional reformat views. It can study and measure arteries from between 0.5 and $18 \mathrm{~mm}$ in diameter and calculate the degree of stenosis. It can display images in a variety of formats, including automatic and simultaneous orthogonal cross-sections, orthogonal MPR, oblique and curved MPR, 3D and curved reformat views [7]. This fast and simple to use software can also be useful for examining the esophageal wall on contrast-enhanced MDCT, clicking on the lesion site. Starting from simple transverse images, VP in MPR mode automatically generates a reference line along the major axis of structures with the maximum enhancement values and displays the best views in multiple curved planes. It also explores the whole thickness of the visceral wall and adjacent structures, which can be useful for preoperative $\mathrm{T}$ staging.

\section{Image Analysis}

Each examination was evaluated independently by two abdominal radiologists (GA, AASI) with more than 10 years experience in abdominal $\mathrm{CT}$ and image reconstruction. They were aware that all patients had proven esophageal cancer, but they were blinded to the endoscopic results, tumor 
location, size and morphological features. A final diagnosis was reached by consensus. The two radiologists evaluated transverse CT images and VP in MPR mode reconstructions in two different sessions, at an interval of 10 days, in a random order. The entire image analysis was performed within 15 minutes per patient. Images were assessed for the presence and site of lesion, thickness, appearance and density of the esophageal wall at the lesion site, depth of visceral wall invasion, morphology of periesophageal fat and infiltration of mediastinal organs. In accordance with literature on CT criteria for $\mathrm{T}$ staging, focal thickening greater than $3 \mathrm{~mm}$ was considered to indicate a neoplastic lesion [9]. Good, intermediate and poor tumor enhancement indicated respectively higher attenuation, isoattenuation and lower attenuation compared with the adjacent normal esophageal wall. $\mathrm{T}$ staging obtained by MDCT was based on the international TNM classification, as shown in Table 1 [9]. MDCT staging was then compared with histological evaluation.

\section{Statistical Analysis}

Sensitivity and diagnostic accuracy values were calculated for axial images alone or in combination with VP in MPR mode reconstructions. The chi-square test was used to compare categorical variables and proportions. A two-tailed probability $(\mathrm{P})$ value of less than 0.05 was considered to indicate a statistically significant difference. In order to evaluate the interobserver agreement between the two readers and the diagnostic reliability of the technique, Cohen's kappa (k) test was used. A $\mathrm{k}$ value of more than 0.81 was considered to represent almost perfect agreement and values of 0.61-0.80 and 0.41-0.60 to represent substantial and moderate agreement, respectively. All calculations were performed using NCSS2007® statistical software. 


\section{Results}

\section{Detection of primary tumor}

All esophageal lesions were detected both in transverse images and VP in MPR mode reconstructions. Neoplasia were identified as pathological wall thickening, measuring between 5.5 and $30 \mathrm{~mm}$ (mean $12 \mathrm{~mm}$ ), with a 10-80 mm longitudinal extension. In 29 out of 31 cases high enhancement was demonstrated at the arterial phase of contrast-enhanced CT examination, while in two patients poor enhancement was found at the lesion site. Eight presented polypoid morphology, 15 infiltrating and 8 presented ulcerative pattern. No case of "early cancer" was detected.

\section{Depth of tumor penetration}

The comparison between $\mathrm{T}$ radiological and histopathological staging is summarised in Table 2 .

In 19 patients who underwent surgical treatment, at histopathological examination 7 were staged as pT2 (fig. 1) and 12 as pT3 (fig. 2). Twelve patients who underwent palliative treatment were staged as T4 because of the invasion of the tracheobronchial tree in 4 cases, aortic invasion in two (fig. 3 ) and tumor extension to the proximal portion of the stomach in the last six cases. Sensibility, negative predictive value (NPV) and overall diagnostic accuracy values of $\mathrm{T}$ staging with axial images were $67 \%, 64 \%$ and $79 \%$, respectively, which improved to $83 \%, 78 \%$ and $89 \%$ with VP in MPR mode reconstructions $(\mathrm{P}<0.05)$. Specificity and positive predictive value (PPV) of $100 \%$ was found for both axial images and VP in MPR mode reconstructions. With transverse images understaging occurred in four patients, whereas with VP images in two cases. Particularly, on transverse images 4 T3 forms were understaged: in 2 cases, irregular outer border of the esophageal wall was not detected; in the remaining 2 patients, small linear strands of soft tissue extending into the periesophageal fat were not recognized. The use of VP in MPR mode reconstructions allowed only 2 T3 carcinomas to be understaged, because of an inhomogeneous and poor enhancement at the lesion sites which did not allow lightly irregular outer border of the esophageal wall to be demonstrated. They were two patients affected by pT3 AEG arising from Barrett's metaplasia with a high mucin content. 
Almost perfect agreement between the two readers was found $(\mathrm{k}=0.83)$.

\section{Discussion}

\section{Diagnostic imaging of esophageal carcinoma}

Esophageal carcinoma has a survival rate of $5 \%$ at 5 years and poor prognosis is due to high infiltrative tendency towards mediastinal organs and high incidence rate of haematic and lymph node metastases $[10,11]$. Accurate preoperative staging of esophageal cancer is essential for the planning of optimal therapy and could influence the survival rate. Therefore, EUS, CT, magnetic resonance imaging (MRI), positron emission tomography (PET) and PET-CT can be performed. EUS allows to depict the internal structure of the esophageal wall, the tumor penetration can be demonstrated with excellent detail and it can guide fine needle aspiration cytology confirmation [12]. In the evaluation of $\mathrm{T}$ parameter, EUS has a diagnostic accuracy rate of $52 \%-92 \%$, but this technique is operator-dependent, with a restricted field of view and it cannot evaluate haematic and lymph node metastases. In addition, often the instrument cannot go beyond lumen strictures caused by tumor growth $[13,14,15]$. MRI presents the same diagnostic capability as CT, although some experiences have reported sensibility and specificity values of $100 \%$ and $84 \%$ respectively for MRI and $100 \%$ and $80 \%$ respectively for CT in the evaluation of the mediastinal invasion $[12,16]$. PET and PET-CT has demonstrated diagnostic accuracy values of 48-90\% in the staging of esophageal cancer. However, these techniques are mostly used in case of advanced forms, especially for evaluating the response to combined chemo- and radiotherapic treatments $[14,17,18]$.

\section{CT staging of esophageal carcinoma}

Despite the advantages of the different techniques, MDCT currently remains the most commonly used examination in preoperative esophageal cancer staging because it allows to rapidly and non invasively evaluate the local extension of the tumor and to detect lymph adenopathies and distant metastases, with overall diagnostic accuracy values of $59-82 \%[6,12,14,19-21]$. In the preoperative staging of T parameter, CT reliability depends on the depth of tumor invasion. In fact, 
CT reliability is lower in detecting stages from T1 to T3 because CT examination does not allow to define intramural invasion, while sensibility values of $83-100 \%$ and $92-100 \%$ have been reported respectively for the definition of tracheo-bronchial tree and aortic-pericardial infiltration [10, 22].

\section{Vessel probe CT protocol}

In our experience, the role of MDCT in the staging of esophageal carcinoma is globally confirmed, but new data emerge from the use of VP in MPR mode reconstructions in the $\mathrm{T}$ staging. This programme allows good detail of the esophageal wall architecture to be obtained after intravenous injection of contrast material and it can improve the diagnostic accuracy in the evaluation of tumor invasion depth by analysing wall enhancement at the lesion site [7]. VP has significantly increased diagnostic accuracy (89\%), sensibility (83\%) and NPV (78\%) in the definition of T parameter compared with CT transverse images (79\%, 67\% and 64\%, respectively) and interobserver variation was low between the two observers, indicating that the reader experience was not a significant parameter and that this technique had good diagnostic reproducibility. In fact, the number of understaging errors has been reduced using VP in MPR mode reconstructions and this software could increase the reliability of MDCT in differentiating T2 from T3 forms [12, 19]. However, the difficulty in distinguishing tumoral invasion of perilesional fat from desmoplastic reaction persists [23]. Besides, obliteration of mediastinal fat layers is reported to be an important CT finding of neoplastic spread beyond the esophageal wall, consequently distinguishing between T3 and T4 forms [5]. In our series, T4 carcinomas have been correctly staged by using both transverse images and VP in MPR mode reconstructions, although it can be supposed that VP could facilitate the detection of advanced forms, thanks to the possibility of immediately and simultaneously examining the contact area between the tumor and mediastinal organs in different axial and longitudinal planes, one perpendicular to each other. Particularly, longitudinal extension of the contact surface between the tumor and aorta could represent a further useful criterion for predicting aortic invasion, in addition to the classic standard reference of measuring contact area in degrees on axial images, especially in case of 45 to $90^{\circ}$ contact surfaces $[20,22,24]$. In fact, the 
greater the craniocaudal extension of the tumor, the more likely it is to be a case of $\mathrm{T} 4$, as already reported for EUS, which predicts advanced carcinomas thanks to longitudinal extensions of more than $5 \mathrm{~cm}$ [25]. Therefore, it can be supposed that VP in MPR mode reconstructions could facilitate the detection of tracheo-bronchial tree invasion, but further research is needed in this field. The use of VP in MPR mode reconstructions only needs the esophageal walls to be adequately distended by air and, as suggested by Umeoka et al [20], an arterial phase at $35 \mathrm{~s}$ to be performed because of the tumor's hypervascularity and neovascularity. In our study protocol, esophagus has been distended by effervescent powder $20 \mathrm{~s}$ before CT scanning in arterial phase and a marked difference in attenuation has been obtained between the lumen and the walls which is essential for the correct application of VP software. In fact, in $94 \%$ of patients high enhancement values have been detected at the lesion sites and it could be supposed that the two understaging errors by VP in MPR mode reconstructions can be due to the low enhancement at the lesion sites which negatively influences image post-processing and probably can vary basing on the different histological patterns. These two cases were demonstrated to be pT3 AEGs arising from distal Barrett's esophagus, which have been already decribed as the most difficult forms to be detected because of a high mucin content and a consequent different enhancing pattern on enhanced MDCT examination compared with SCCs [7, 20]. In conclusion, 16 row MDCT represents an accurate tool in the preoperative T staging of esophageal carcinoma. In case of T2 and T3 esophageal carcinoma, the use of fast, easy and automatic VP in MPR mode reconstructions increase the diagnostic accuracy compared with transverse images for the evaluation of local extension of the disease which is crucial for the choice of surgical or alternative treatments.

\section{Limitations}

However, our study has some limitations represented by the short number of studied patients; absence of T1 forms and impossibility of having a comparison between CT and EUS in T2 and T3 staging. Therefore, further studies with larger number of patients and CT systems with more than 16 or 64 detectors are needed in this field, also to research if VP algorithm could be useful in 
detecting early cancer and to evaluate the response to chemo-radiotherapy in patients with advanced forms.

\section{References}

1. Brown LM, Devesa SS, Chow WH. Incidence of adenocarcinoma of the esophagus among white Americans by sex, stage, and age. J Natl Cancer Inst 2008; 100:1184-7.

2. Parker SL, Tong T, Bolden S, Wingo PA. Cancer Statistics. CA Cancer J Clin 1997; 47:5-27.

3. Bollschweiler E, Wolfgarten E, Gutschow C, Hölscher AH. Demographic variations in the rising incidence of esophageal adenocarcinoma in white males. Cancer 2001; 92:549-55.

4. Yousef F, Cardwell C, Cantwell MM. The incidence of esophageal cancer and highgrade dysplasia in Barrett's esophagus: a systematic review and meta-analysis. Am J Epidemiol 2008; 168:237-49.

5. Fiore D, Baggio V, Ruol A, et al. Multimodal imaging of esophagus and cardia cancer before and after treatment. Radiol Med 2006; 111:804-17.

6. Panebianco V, Grazhdani H, Iafrate F, et al. 3D CT protocol in the assessment of the esophageal neoplastic lesions: can it improve TNM staging? Eur Radiol 2006; 16: 41421.

7. Moschetta M, Stabile Ianora AA, Anglani A, et al. Preoperative T staging of gastric carcinoma obtained by MDCT vessel probe reconstructions and correlations with histological findings. Eur Radiol 2010; 20: 138-45.

8. Green FL, Page DL, Fleming ID, et al. Esophagus. In: Springer-Verlag ed. AJCC cancer staging manual, New York; 2002, p 105. 
9. Ba-Ssalamah A, Zacherl J, Noebauer-Huhmann IM, et al. Dedicated multi-detector CT of the esophagus: spectrum of diseases. Abdom imaging 2009; 34:3-18.

10. Wayman J, Chakraverty S, Griffin SM, et al. Evaluation of local invasion by esophageal carcinoma: prospective study of prone computed tomography scanning. Postgrad Med J $2001 ; 77: 181-4$.

11. Holscher AH, Dittler HJ, Siwert JR. Staging of squamous esophageal cancer: accuracy and value. World J Surg 1994; 18:312-20.

12. Wu LF, Wang BZ, Feng JL, et al. Preoperative TN staging of esophageal cancer: comparison of miniprobe ultrasonography, spiral CT and MRI. World J Gastroenterol 2003; 9:219-24.

13. Ligresti R, Lightdale CJ. Diagnosis of esophagogastric tumours. Endoscopy 1998; 30:73-9.

14. Sandha GS, Severin D, Postema E, McEwan A, Stewart K. Is positron emission tomography useful in locoregional staging of esophageal cancer? Results of a multidisciplinary initiative comparing $\mathrm{CT}$, positron emission tomography and EUS. Gastrointest Endosc 2008; 67:402-9.

15. Munro AJ. Oesophageal cancer: a view over overviews. Lancet 2004; 364:566-8.

16. Takashima S, Takeuchi N, Shiozaki H, et al. Carcinoma of the esophagus: CT vs MR imaging in determining respectability. Am J Roentgenol 1991; 156:297-302.

17. Vinjamuri S, Ray S. Added value of PET and PET-CT in oesaophageal cancer: a review of current practice. Nucl Med Commun 2008; 29:4-10.

18. Bruzzi JF, Munden RF, Truong MT, et al. PET-CT of esophageal cancer: its role in clinical management. Radiographics 2007; 27:1635-52.

19. Weaver S, Blackshaw G, Lewis W. Comparison of special interest computed tomography, endosonography and histopathological stage of oesophageal cancer. Clin Radiol 2004 ; 59:499-504. 
20. Umeoka S, Koyama T, Togashi K, et al. Esophageal Cancer: Evaluation with TriplePhase Dynamic CT- Initial Experience. Radiology 2006; 239:777-83.

21. Bonavina L, Incarbone R, Saino G, Clesi P, Peracchia A. Clinical outcome and survival after esophagectomy for carcinoma in elderly patients. Dis Esophagus 2003; 16:90-3.

22. Kumbasar B. Carcinoma of esophagus: radiologic diagnosis and staging. Eur J Radiol 2002; 42:170-80.

23. Korst RJ, Altorki NK. Imaging for esophageal tumours. Thorac Surg Clin 2005; 14:619.

24. Iyer R, Dubrow R. Imaging of esophageal cancer. Cancer Imaging 2004; 4:125-32.

25. Bhutani MS, Barde CJ, Market RJ, Gopalswamy N. Length of esophageal cancer and degree of luminal stenosis during upper endoscopy predict $\mathrm{T}$ stage by endoscopic ultrasound. Endoscopy 2002; 34:461-3. 


\section{Table 1.}

Pathologic TNM staging system developed by the American Joint Committee on Cancer (2002) and MDCT Criteria for T staging of gastric cancer (2009).

\section{Table 2.}

MDCT findings compared with histopathological results for each T stage.

\section{Figure 1.}

Intramural carcinoma of the esophageal lower third in a 46-year-old woman (pT2). a) Transverse CT scan demonstrates eccentric thickening of the esophageal wall (arrowhead) and cleavage from the surrounding structures. VPs in MPR mode reconstructions allow better evaluation of triangular fat space between esophageal lesion site, aorta and spine, suggesting no periesophageal fat invasion (arrows). b) The histopathological specimen shows a scarcely differentiated squamous cell carcinoma which reaches the muscolaris propria layer: T, tumor; M: muscolaris propria layer (hematoxylin-eosin stain, x 100 magnification).

\section{Figure 2.}

Extramural carcinoma of the esophageal upper third in a 62 year-old-woman (pT3). Transverse CT scan shows concentric esophageal wall thickening (arrowhead) involving perilesional fat. VPs in MPR mode reconstructions can better evaluate perilesional fat tissue hyperdensity and linear strands (arrows). b) The histopathological specimen shows a scarcely differentiated squamous cell carcinoma which invades adventitia: T, tumor; A: adventitia (hematoxylin-eosin stain, x 100 magnification).

\section{Figure 3.}

Esophageal carcinoma located at the middle third in a 65 year-old-man (T4). Transverse CT scan demonstrates irregular concentric thickening of the esophageal walls (arrowhead). Tumor invades the aorta with a circumferential adhesion area of more than $90^{\circ}$. VPs in MPR mode reconstructions confirm this finding in multiple oblique and longitudinal planes with a better evaluation of the contact area's extension between tumor and the aorta (arrows). 
Conflict of interest statement. The authors declare that they have no conflict of interest to the publication of this article. 


\begin{tabular}{|c|l|}
\hline pT1 & \multicolumn{1}{|c|}{ HISTOLOGICAL TNM and MDCT Criteria } \\
T1 & $\begin{array}{l}\text { Tumour invades lamina propria or submucosal layer } \\
\text { enhancement only without wall thickening, or thickening and marked enhancement } \\
\leq 5 \text { mm in diameter }\end{array}$ \\
\hline pT2 & $\begin{array}{l}\text { Tumour invades muscolaris propria } \\
\text { Transmural enhancement with focal wall thickening between 5 and 15 mm in } \\
\text { Tiameter; smooth outer border of the thickened esophageal wall, or a few small linear } \\
\text { strands of soft tissue extending into the fat plane, but less than one-third of the tumor } \\
\text { extension associated with no or slight stenosis }\end{array}$ \\
\hline pT3 & $\begin{array}{l}\text { Tumour invades adventitia } \\
\text { Usually large tumor more than } 10 \text { mm in depth, with reticular or irregular outer } \\
\text { border of the thickened esophageal wall, or stranding and densities more than one- } \\
\text { third of the tumor extension or a blurred fat plane around the lesion associated with } \\
\text { moderate to severe stenosis }\end{array}$ \\
\hline pT4 & $\begin{array}{l}\text { Tumour invades adjacent structures } \\
\text { Gross infiltration of adjacent organs }\end{array}$ \\
\hline
\end{tabular}

\section{Table 1.}

Pathologic TNM staging system developed by the American Joint Committee on Cancer (2002) and MDCT Criteria for T staging of gastric cancer (2009). 
Table 2

\begin{tabular}{|c|c|c|c|}
\hline & pT2 n=7 & pT3 n=12 & pT4=12 \\
\hline Axial & \multicolumn{3}{|c|}{} \\
\hline T2 & 7 & 4 & \\
\hline T3 & & 8 & 12 \\
\hline T4 & & \multicolumn{1}{l|}{} \\
\hline VP in MPR mode reconstructions & \\
\hline T2 & 7 & 12 & 12 \\
\hline T3 & & 10 & \\
\hline T4 & & & \\
\hline
\end{tabular}

MDCT findings compared with histopathological results for each $\mathrm{T}$ stage. 


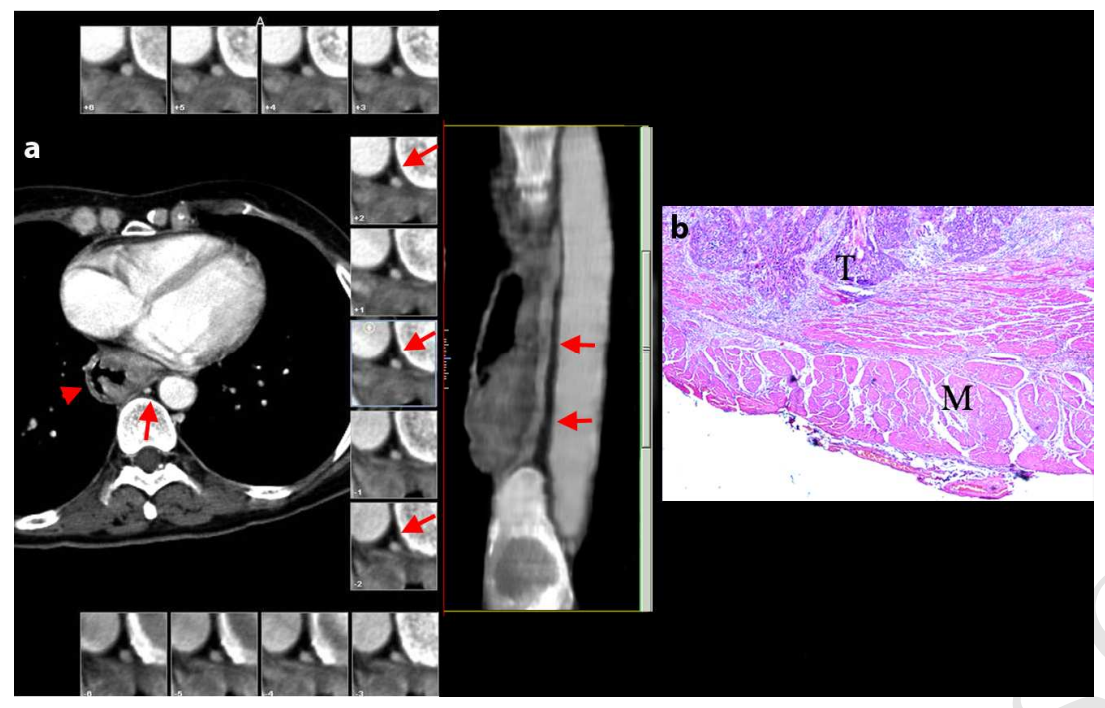




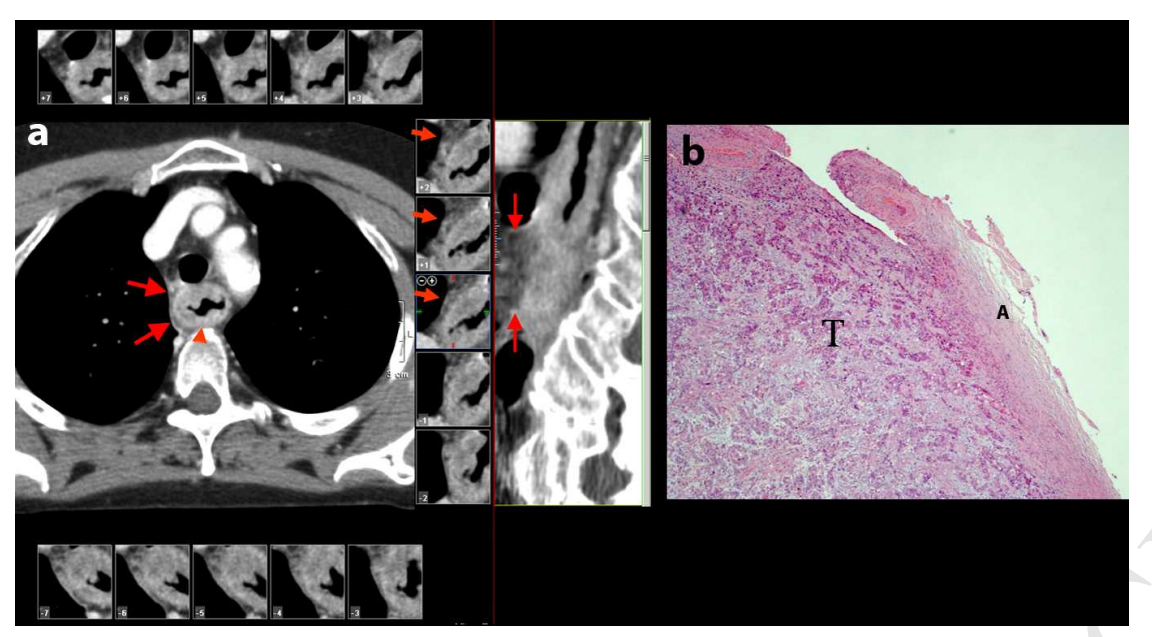




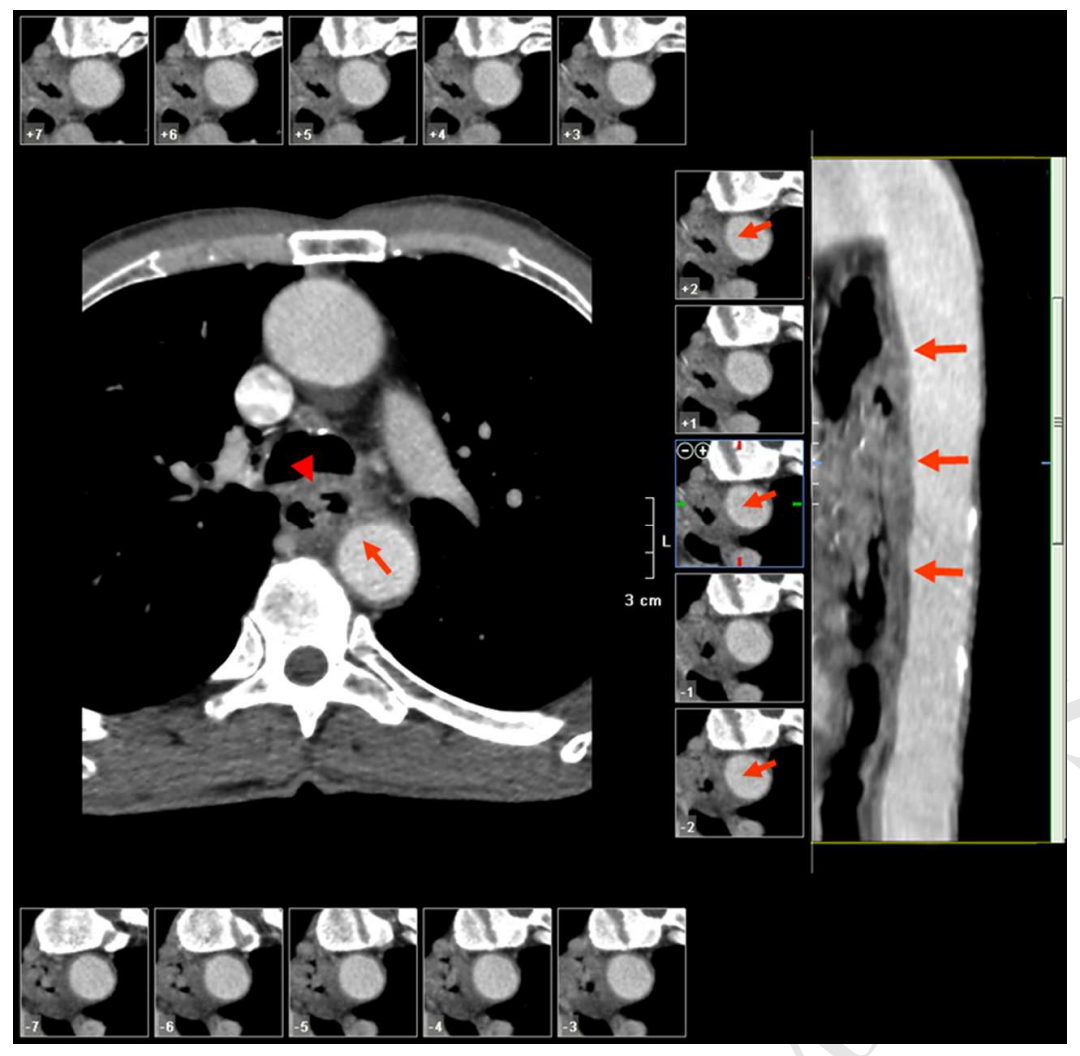

